\title{
Recovery of the grain boundary character distribution through oblique double-sectioning
}

\author{
Brent L. Adams \\ b_I_adams@byu.edu \\ David T. Fullwood \\ dfullwood@byu.edu \\ E. R. Homer
}

Follow this and additional works at: https://scholarsarchive.byu.edu/facpub

Part of the Mechanical Engineering Commons

Original Publication Citation

Scripta Materialia 54 (26) 117-121

\section{BYU ScholarsArchive Citation}

Adams, Brent L.; Fullwood, David T.; and Homer, E. R., "Recovery of the grain boundary character distribution through oblique double-sectioning" (2005). Faculty Publications. 339.

https://scholarsarchive.byu.edu/facpub/339 


\title{
Recovery of the grain boundary character distribution through oblique double-sectioning
}

\author{
E.R. Homer *, B.L. Adams, D.T. Fullwood \\ Department of Mechanical Engineering, Brigham Young University, 435 CTB, Provo, UT 84602, USA
}

\begin{abstract}
A method for the retrieval of the complete grain boundary character distribution by oblique double-sectioning is proposed. The method, which is similar to the recovery of the orientation distribution from sets of incomplete pole-figures, is efficient and provides many advantages as compared to calibrated serial sectioning. As compared to standard stereological approaches, the new methodology retains the advantage of direct measurement of the grain boundary inclination parameters. Solutions to the fundamental equation of oblique double-sectioning are provided in the Fourier space, and some specifics in sample preparation are introduced.
\end{abstract}

Keywords: Grain boundary structure; Microstructure; Orientation imaging microscopy (OIM)

\section{Introduction}

The interfacial characteristics of polycrystalline materials often have a large effect upon the macroscopic properties of a material. This is due in large part to the crystallographic nature of the interfaces that gives rise to substantial variability in the intrinsic properties of boundaries $[1,2]$. In single phase polycrystals the connectivity and distribution among crystallographic types of these interfaces, or grain boundaries, requires adequate representation. Of central interest here is one of the most commonly employed representations, the grain boundary character distribution (GBCD) [3].

The ability to recover the GBCD with minimal time and effort is of prime importance in grain boundary engineering (GBE) because GBE focuses on optimizing the macroscopic properties of a material by altering certain characteristics of the GBCD. This article points to new methodology for the recovery of the GBCD and compares it with other available methods.

\footnotetext{
* Corresponding author. Tel.: +1 801422 2625; fax: +1 8014220516. E-mail address: eric.homer@byu.edu (E.R. Homer).
}

The GBCD requires specification of eight macroscopic (crystallographic) parameters for proper representation when grain boundaries interact with external fields. This reflects the fact that a crystalline grain requires three parameters to define its lattice orientation. Thus, for a grain boundary separating two grains, six parameters are required for orientation purposes while two additional parameters are necessary to describe the inclination of the grain boundary. The GBCD can be reduced to five parameters when represented in the local crystal frame since only three parameters are needed to define the misorientation between the grains and the tie to the specimen frame is no longer necessary [3].

In the past it has proven difficult to recover the two parameters of the GBCD representing the boundary inclination. While the direct stereological methods of Hilliard [4] and Adams [5] enable recovery of the inclination parameters of the GBCD via the solution to inverse problems using the method of Fourier series, these methods can be mathematically cumbersome. And in the case of the Hilliard methodology, only the even components of the distribution of inclination parameters can be recovered. A summary of these methods, comparisons between them, and discussion of their limitations is given by Adams et al. 
[3]. As a result of these complexities, many early investigations into the GBCD focused only on the misorientation of the grain boundary, which is readily available by automated scanning electron (diffraction) microscopy, commonly known as orientation imaging microscopy (OIM). This is noted by the abundance of investigations focused on coincidence site lattice (CSL) models [6,7]. In general CSL boundaries have been shown to exhibit special properties and for this reason the boundary recognition schemes relying heavily upon CSL boundaries have proliferated. Despite limited success, properties predictions based solely on misorientation parameters have not always performed as predicted and non-CSL boundaries have exhibited special properties in some cases [8]. This lack of consistency between the predicted and actual performances of certain boundary types can be attributed, in part, to the inclination of the boundary that is not measured in the previous CSL studies [8,9]. In fact the behavior of the boundary may be more sensitive to the boundary plane orientation than the grain misorientation [10].

\section{Serial sectioning}

In order to recover the full GBCD, including boundary inclination, one may employ the well-established method of serial sectioning. Full three-dimensional microstructures can be reconstructed from a sample by repeating the following process: remove a thin layer of material from the sample after which the sample surface is scanned using OIM. The OIM scans obtained from each layer of the sample contain the necessary crystallographic orientation data but the boundary inclination is still missing from the GBCD. The boundary inclinations of all the grain boundaries are approximated by triangulating a group of adjacent OIM data points using a meshing algorithm [11]. An entire grain boundary is therefore given by a conglomeration of small polygons, which are created by superimposing

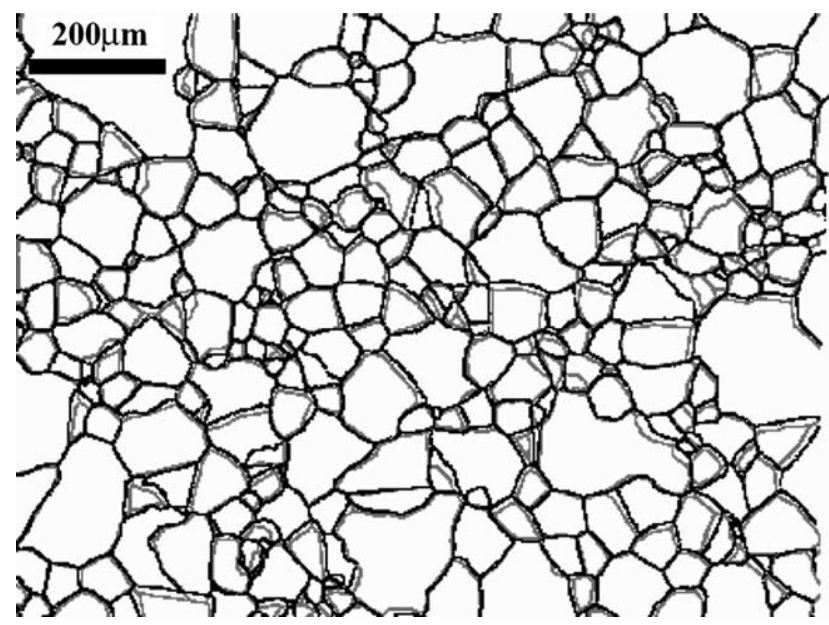

Fig. 1. The superposition of boundaries obtained from two separate OIM scans after being aligned. (Figure used with permission [11].) two layers of the serial section data at a time, as seen in Fig. 1. The meshing algorithm, which is illustrated in Fig. 2, first connects data points representing triple junctions after which the nearest vertex position on the opposing layer is connected to this set to form a surface or polygon. The next nearest neighbor is then connected to this set to form another surface or polygon. This process is repeated until all data points on the two layers are connected. In this manner, one can obtain the boundary inclination for all the grain boundaries represented by the two layers of OIM data [11].

One very important element to the method of serial sectioning is that of aligning the different layers of OIM data. This is most often done by micro-hardness indents but can also be done with specific microstructural features such as grain boundary triple junctions located at the base of stable twins [8]. In any case, care must be taken while aligning the sections because of the error that can be introduced if performed incorrectly. Another element that must be accounted for with great accuracy is the amount of material which is removed before each subsequent scan. This can also be measured using the micro-hardness indents or by precision, calibrated removal of the material under a determined load for a specific amount of time. When properly conducted, the inclinations of the boundaries can be determined with $\sim 5^{\circ}$ of precision [10].

(a)

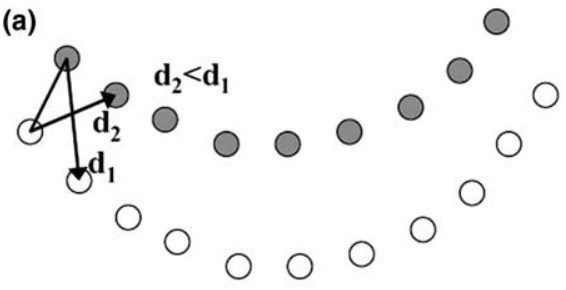

(b)

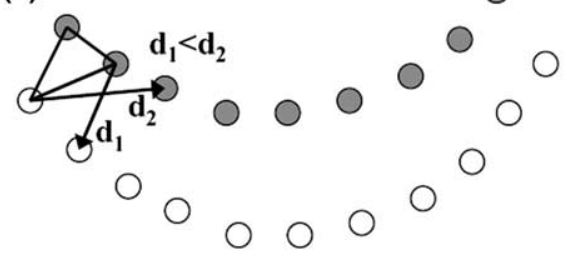

(c)

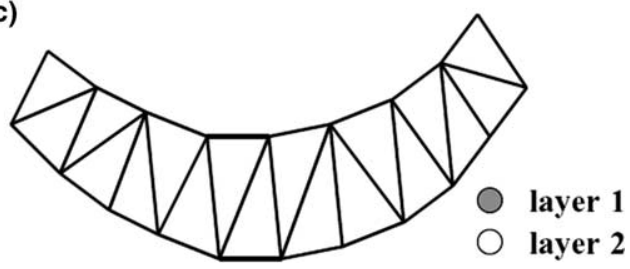

Fig. 2. Grain boundary meshing algorithm. (a) After OIM datapoints are projected onto opposite layers grain boundary triple junctions are connected after which the next nearest point on the opposing layer is connected to this group to form a polygon. (b) This process is repeated to form the next polygon and so on until all points have been connected. (c) All grain boundaries in the two layers are represented as a conglomeration of small polygons. (Figure used with permission [11].) 
Serial sectioning is a tedious process that requires a great amount of precision, skill and time to produce any volume of information from a sample. Although not limited to this range, anywhere from 50 to 150 section planes may be desired to obtain suitable information about the geometry of the grains, this number being dependent upon the sample removal thickness and other geometrical features of the material $[12,13]$. Finally, the volume of the sample being tested with serial sectioning originates from a single position in the material and therefore may not be representative of the bulk material.

\section{Oblique double-sectioning}

In this article, we describe how an adaptation of the method of serial sectioning can be used to obtain the full GBCD with less effort. In the proposed method of oblique double-sectioning (ODS) a sample will be cut into a number of sections at oblique angles. Each of these section-cuts will then be scanned with OIM after which one layer will be removed and the sample will be scanned again. In contrast to serial sectioning, only two OIM scans will be recovered from each oblique section plane. The meshing algorithm described above is then used to get the boundary inclinations of each of the grain boundaries. In this manner, the GBCD, although incomplete, can be recovered for each of the double-sections. Then, according to a method which will be presented hereafter, the full GBCD for the material can be recovered.

As an artifact of ODS, data for the inclination of boundaries in each section plane are incomplete. It is presumed that the inclination of the boundary, $\hat{n}$, is expressed in a specified local orthonormal sectioning frame, say $\left\{{ }^{(c)} \hat{e}_{i}\right\}$, where the spherical-polar angles associated with $\hat{n}$ in the sectioning frame, $c$, are $\phi^{\prime}, \theta^{\prime}$. The degree to which the data is incomplete is dependent upon the grain size and the amount of material removed between the two layers; specifically $\hat{n}$ is only available over the range $\phi_{a}^{\prime} \leqslant \phi^{\prime} \leqslant \pi / 2,0 \leqslant \theta^{\prime}<2 \pi$, where

$\phi_{a}^{\prime} \approx \frac{\text { section removal thickness }}{\text { grain size }}$.

This is due to the potential for boundaries of both small and large grains alike to go unobserved by not appearing in either of the section planes.

To overcome this artifact, multiple section-cuts are taken at oblique angles from a large sample to form an ensemble of samples. This is done in a manner so as to cover a hemisphere of section normal directions as uniformly as possible such that the hole in the data from any one section-cut will be covered by the overlap of data from other section-cuts. In general the number of sectioncuts required to recover the overall GBCD will be dependent upon both the grain size of the material as well as the sample removal thickness. Increasing the number of section-cuts increases the accuracy of the method but this comes at the cost of extra sample preparation time.

\section{Preparation of oblique section-cuts}

While ODS may be performed with as few as three orthogonal section-cuts we present here a scheme where 13 section-cuts are prepared in such a manner that their section normals cover a hemisphere of directions as uniformly as possible. The section normals for this scheme are shown in Fig. 3 where each of the section-cuts is numbered. The figure also shows the inverse of each of the normals and for clarity, points on the back side of the hemisphere are shown in gray while points on the front are shown in black. In order to prepare the samples with the normals given in Fig. 3 a possible cutting scheme is presented in Fig. 4 where the normal of each section-cut is given by the numbered direction shown in Fig. 3. In this manner, the samples can be prepared with relative ease; although one may choose to perform a similar process but with a greater or lesser number of section-cuts.

\section{Recovery of the GBCD}

The GBCD of the material can now be obtained from the OIM data of the sets of oblique double-sections in the following manner. In presenting the ODS method, for pedagogical clarity we will only consider the simplified GBCD, which carries only the grain boundary normal distribution $S_{V}(\hat{n})$, and let it accept a finite Fourier representation of the form

$S_{V}(\hat{n})=\sum_{l=0}^{\infty} \sum_{m=-l}^{+l} S_{l}^{m} k_{l}^{m}(\hat{n})$.

The $S_{l}^{m}$ coefficients will be determined from the OIM data of the oblique double-sections and the $k_{l}^{m}(\hat{n})$ functions

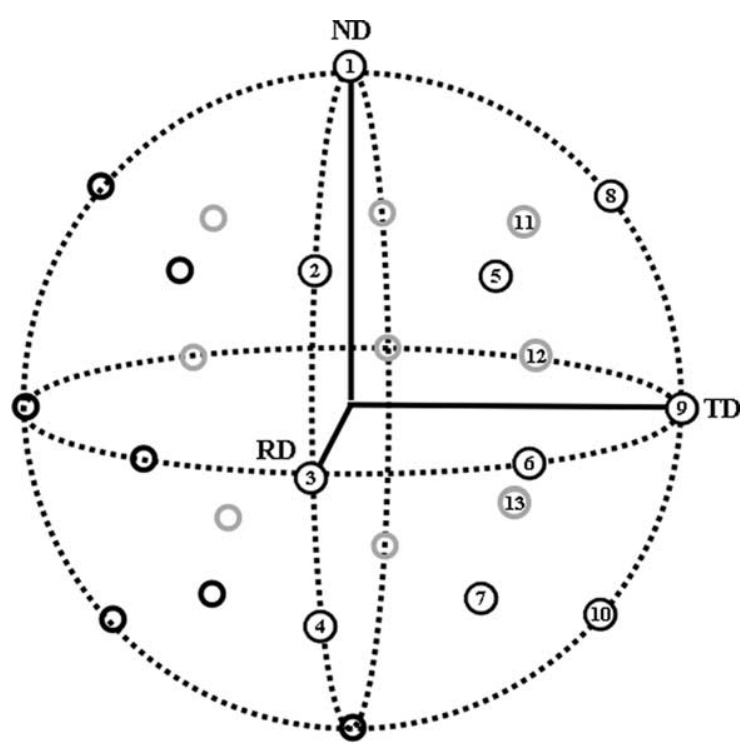

Fig. 3. Sphere of directions representing the normal directions for 13 different section-cuts along with their inverse. Points on the front half of the sphere are shown in black while points on the back half are shown in gray. 


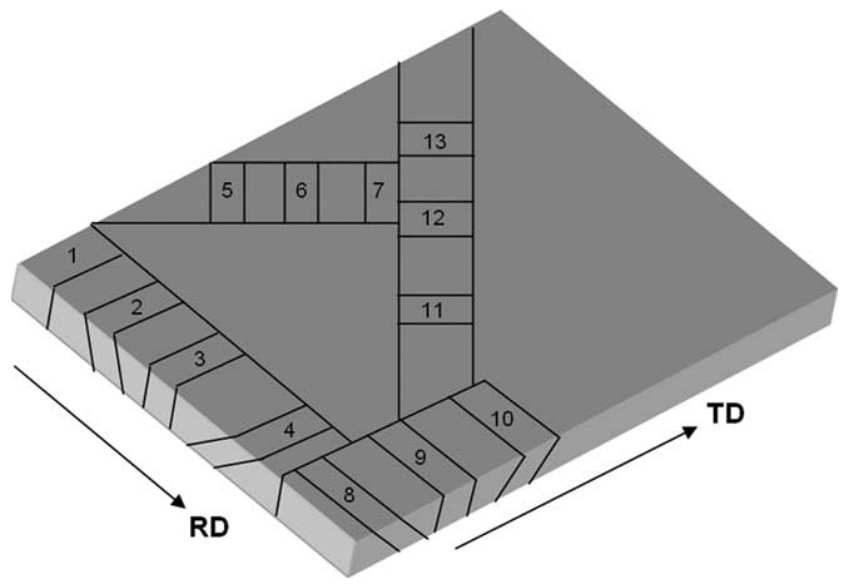

Fig. 4. Representation of possible cutting scheme, where 13 samples will be cut from one specimen to be examined by oblique double-sectioning. Numbered section-cuts correspond to the normal directions shown in Fig. 3.

are the classical surface spherical harmonic functions [14]. As there are several different oblique double-sections, each one will be enumerated by the letter $c$. The $c$ th coordinate frame is related to the specimen frame, $\left\{\hat{e}_{i}\right\}$, by rotation of ${ }^{(c)} g^{-1}$ to become $\left\{{ }^{(c)} \hat{e}_{i}\right\}$. Here the notation ${ }^{(c)} g^{-1}$ is used to emphasize the fact that Bunge's notation is in terms of the direction cosines, and these are related to the rotation tensor by inversion [14]. The spherical-polar angles associated with the new $c$ th frame are $\phi^{\prime}, \theta^{\prime}$. It can then be shown that the coefficients in the $c$ th coordinate frame are related to the specimen frame by

${ }^{(c)} S_{l}^{\mu}=\sum_{m=-l}^{+l} S_{l}^{m} T_{l}^{m \mu}\left({ }^{(c)} g^{-1}\right)$,

where $T_{l}^{n m}(\mathrm{~g})$ are the classical generalized spherical harmonic functions [14].

The problem of recovery of the best set of Fourier coefficients $S_{l}^{m}$ can then be posed in a manner similar to the recovery of orientation distribution functions from incomplete pole-figures as described by Bunge [14]. Consider the measured difference between the true GBCD in the $c$ th section plane, say ${ }^{(c)} S_{V}\left(\phi^{\prime}, \theta^{\prime}\right)_{\text {cal. }}$, and the observed distribution, ${ }^{(c)} S_{V}\left(\phi^{\prime}, \theta^{\prime}\right)_{\text {obs. }}$, within the allowable region of observations, to be

$\int_{\phi_{a}^{\prime}}^{\pi / 2} \int_{0}^{2 \pi}\left[{ }^{(c)} S_{V}\left(\phi^{\prime}, \theta^{\prime}\right)_{\text {obs. }}-{ }^{(c)} S_{V}\left(\phi^{\prime}, \theta^{\prime}\right)_{\text {cal. }}\right]^{2} \sin \phi^{\prime} \mathrm{d} \phi^{\prime} \mathrm{d} \theta^{\prime}$.

This difference in the observed and calculated GBCD can be minimized if it is assumed that the accuracy of the data from each section-cut is equal and if it is recognized that the observed data on each section-cut may have different normalization factors, $\eta_{c}$. With these assumptions the following minimization problem emerges

$\sum_{c=1}^{N_{c}} \iint_{B}\left[\eta_{c}{ }^{(c)} S_{V}\left(\hat{n}^{\prime}\right)_{\text {obs. }}-\sum_{l=0}^{\infty} \sum_{m=-l}^{+l} S_{l}^{m(c)} \xi_{l}^{m}\left(\hat{n}^{\prime}\right)\right]^{2} \mathrm{~d} \hat{n}^{\prime}=\min$, where

$$
\begin{aligned}
& B=\left\{\hat{n}^{\prime} \equiv\left(\phi^{\prime}, \theta^{\prime}\right) \mid \phi_{a}^{\prime} \leqslant \phi^{\prime} \leqslant \pi / 2,0 \leqslant \theta^{\prime}<2 \pi\right\}, \\
& \mathrm{d} \hat{n}^{\prime}=\sin \phi^{\prime} \mathrm{d} \phi^{\prime} \mathrm{d} \theta^{\prime}
\end{aligned}
$$

and

${ }^{(c)} \xi_{l}^{m}\left(\hat{n}^{\prime}\right)=\sum_{\mu=-l}^{+l} T_{l}^{m \mu}\left({ }^{(c)} \mathrm{g}^{-1}\right) k_{l}^{\mu}\left(\hat{n}^{\prime}\right)$

The representation of Eq. (5) is convenient because it allows a best least-squares fit to be determined by choosing the variables $\eta_{c}$ and $S_{l}^{m}$. Therefore, if Eq. (5) is differentiated with respect to both $S_{l^{\prime}}^{m^{\prime}}$ and $\eta_{c^{\prime}}$ and a substitution is subsequently made for $\eta_{c}$ the following equality is obtained

$\sum_{l=0}^{\infty} \sum_{m=-l}^{+l} S_{l}^{m} \chi_{l l^{\prime}}^{m m^{\prime}}=0$

where

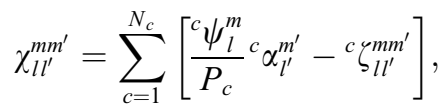

in terms of the reduced variables

$P_{c}=\iint_{B}\left({ }^{(c)} S_{V}\left(\hat{n}^{\prime}\right)_{\text {obs. }}\right)^{2} \mathrm{~d} \hat{n}^{\prime}$,

${ }^{c} \alpha_{l^{\prime}}^{m^{\prime}}=\iint_{B}{ }^{(c)} S_{V}\left(\hat{n}^{\prime}\right)_{\text {obs. }}{ }^{(c)} \xi_{l^{\prime}}^{m^{\prime}}\left(\hat{n}^{\prime}\right) \mathrm{d} \hat{n}^{\prime}$,

${ }^{c} \psi_{l}^{m}=\iint_{B}{ }^{(c)} S_{V}\left(\hat{n}^{\prime}\right)_{\mathrm{obs} .}{ }^{(c)} \xi_{l}^{m}\left(\hat{n}^{\prime}\right) \mathrm{d} \hat{n}^{\prime}$

and

${ }^{c} \zeta_{l l^{\prime}} m^{\prime}=\iint_{B}(c) \xi_{l^{\prime}}{ }^{\prime}\left(\hat{n}^{\prime}\right)^{(c)} \xi_{l}^{m}\left(\hat{n}^{\prime}\right) \mathrm{d} \hat{n}^{\prime}$.

As has been shown, the $\chi_{l l^{\prime}}^{m m^{\prime}}$ coefficients are all in terms of the measured GBCD data obtained from the different oblique double-sections. In examining Eq. (9) further, one will notice that it is a homogeneous set of equations where the number of equations is exactly equal to the number of unknowns. One method of dealing with the homogeneous system of equations is to add one more condition obtained by measuring the global average value of $S_{V}$ (measured using standard OIM methods)

$\bar{S}_{V}=\iint_{S^{2}} S_{V}(\hat{n}) \mathrm{d} \hat{n}=\sum_{l=0}^{\infty} \sum_{m=-l}^{+l} S_{l}^{m} \iint_{S^{2}} k_{l}^{m}(\hat{n}) \mathrm{d} \hat{n}$.

The resulting set of equations for the $S_{l}^{m}$ coefficients is overconstrained, but inhomogeneous, and can be solved using standard techniques. Alternatively, if the normalization factors, $\eta_{c}$, are known the derivation would proceed in a slightly different manner resulting in a system of inhomogeneous equations that may be solved without the addition of 
the extra condition. In either manner one can recover the least-squares best fit of the GBCD in direct space by ODS.

\section{Discussion of oblique double-sectioning}

ODS is expected to have several advantages relative to other stereological methodologies and comprehensive calibrated serial sectioning, in recovering the GBCD.

- Comparison with $P_{L} / S_{V}$ stereology of Hilliard [4] and $L_{A} / S_{V}$ stereology of Adams [5]

- ODS directly measures the inclination parameters of the GBCD.

- Stereological methods require solution to inverse problems, which are known to suffer from certain ambiguities and approximations [3].

- Comparison with comprehensive calibrated serial sectioning.

- Experimental cost of ODS is expected to be reduced substantially.

- ODS provides better representation of bulk material properties by sampling an ensemble of section-cuts from the material.

- The effect of incomplete datasets that result from the finite material removal process between sections will be reduced for ODS as a result of the ensemble of oblique section-cuts when compared with the set of parallel section-cuts of serial sectioning.

- The potential experimental error associated with the alignment of a series of section-planes associated with serial sectioning is reduced by the need to align only two section-planes on each section-cut for ODS.

- Boundary inclination measurement error may be increased for ODS since only two section-planes are used to measure a boundary when compared with multiple section-planes measuring a single boundary in serial sectioning.

- Three-dimensional connectivity information is lost with the ODS methodology which is otherwise available via comprehensive serial sectioning.

\section{Summary and conclusions}

The paper proposes a new methodology for recovery of the full GBCD from quasi-three-dimensional experimental data sets. Called oblique double-sectioning, the method is similar to the problem of recovering the orientation distribution function from sets of incomplete pole-figures. The problem that is overcome is the incompleteness of pole-distributions of grain boundary data arising from the ratio of grain size to section thickness. Mathematical methodology is described for recovering the least-squares best fit to the GBCD for a given set of ODS data. The anticipated advantages of ODS, relative to available stereological methods and comprehensive serial sectioning, were described. With respect to common two-dimensional analyses focused only upon misorientation features, ODS is capable of recovering all eight macroscopic parameters of the GBCD.

\section{Acknowledgement}

This work was supported primarily by the MRSEC program of the National Science Foundation under DMR-0079996.

\section{References}

[1] Sutton AP, Balluffi RW. Interfaces in crystalline materials. Oxford: Clarendon Press; 1995.

[2] Wolf D, Yip S, editors. Materials interfaces: atomic-level structure and properties. London: Chapman \& Hall; 1992.

[3] Adams BL, Field DP. Metall Trans A 1992;23A:2501.

[4] Hilliard JE. Trans AIME 1962;224:1201.

[5] Adams BL. Metall Trans A 1986;17A:2199.

[6] Crawford DC, Was GS. Metall Trans A 1992;23A:1195.

[7] Palumbo G, Aust KT. Acta Metall Mater 1990;38:2343.

[8] Henrie A, Adams BL. Metall Mater Trans, submitted for publication.

[9] Pan Y, Adams BL, Olson T, Panayotou N. Acta Mater 1996;44:4685.

[10] Rohrer GS, Saylor DM, El-Dasher B, Adams BL, Rollett AD, Wynblatt P. Z Metallkd 2004;95:197.

[11] Saylor DM, Morawiec A, Rohrer GS. Acta Mater 2003;51:3663.

[12] DeHoff RT, Aigeltinger EH, Craig KR. J Microsc 1972;95:69.

[13] DeHoff RT. J Microsc 1983;131:259.

[14] Bunge H-J. Texture analysis in materials science. Göttingen: Cuvillier Verlag; 1993. 